\title{
ARQUITECTURA Y ESTUDIOS URBANOS EN LA COMPLEJIDAD DEL SIGLO XXI LA FAU-UCE DESDE EL SUR
}

\section{ARCHITECTURE AND URBAN STUDIES IN THE COMPLEXITY OF THE 21ST CENTURY THE FAU-UCE FROM THE SOUTH}

(iD) Rosa Mishell Echeverría Bucheli ${ }^{1 *}$, (D) Sergio Andrés Bermeo Álvarez ${ }^{1}$

rmecheverria@uce.edu.ec; sabermeo@uce.edu.ec

1Universidad Central del Ecuador, Quito, Ecuador

*Correspondencia: Rosa Mishell Echeverría Bucheli. Email: rmecheverria@uce.edu.ec

Recibido: 11.08.21 | Aprobado: 28.08.21

\section{RESUMEN}

En la actualidad la arquitectura enfrenta un proceso de globalización con patrones que están cambiando las estructuras urbanas, sociales, económicas y culturales hacia el paradigma de la complejidad, con dinámicas en constante interacción, resultando para los estudios urbanos un conjunto intrincado y difícil de comprender. Este cambio requiere una visión sistémica para reconocer tanto las propiedades del objeto, como el comportamiento de los sujetos en torno a un sistema arquitectónico, que en conjunto suman más que el todo. Es importante la reflexión desde la academia, asumiendo una enseñanza interdisciplinar y con una visión epistemológica desde el sur global.

Palabras clave: Arquitectura, complejidad, enseñanza-aprendizaje

\begin{abstract}
The objective was to analyze the reality of the pedagogical practice and to know what has been the turn that the pandemic has caused in the institutions of secondary education; To achieve this, the quantitative methodology was used with the application of descriptive statistics. Evidence that $66.67 \%$ do not agree to integrate the methodological strategies of technological pedagogy into their pedagogy while $77.78 \%$ claim not to assume the new pedagogical methods within the formative dynamics. It was concluded that the pedagogy developed is attached to the face-to-face paradigm only with the change in resources and means. The complexity of the situation is more related to the willingness and interest of the institutions that must internalize the achievement of educational quality through innovation.
\end{abstract}

Keywords: Architecture, complexity, teaching-learning 


\section{INTRODUCCIÓN}

En el siglo XXI, las ciudades latinoamericanas empiezan a evidenciar cambios territoriales producto de: la globalización, nuevas tecnologías de información y comunicación, reestructuración socio-económica, políticas urbanas neoliberales, crecimiento demográfico y estructura urbana, expansión metropolitana, así como, peri urbanización (de Mattos, 1998); (Sassen, 1994); (Parnreiter, 2005); (Borja, 2003). Estas dinámicas que responden a la crisis de la modernidad, e impactan también a la arquitectura y su enseñanza, son el reflejo de la aceleración de los flujos de capital económico, humano y tecnológico para privilegiar al nuevo poder del mercado neoliberal, es decir, bajo la lógica de acumulación las áreas metropolitanas sobreponen un neourbanismo tardorracionalista capitalista, que transforma la arquitectura de la ciudad en fronteras difusas e interconectadas (Bermeo, 2019).

En la actualidad aparece un nuevo paradigma que responde a la teoría de la complejidad o más conocido como pensamiento complejo (Morin, 2004), a la que la arquitectura y los estudios urbanos deben acogerse, para entender cómo la arquitectura, la ciudad y la sociedad, se desarrollan bajo sistemas complejos que reemplazan la visión de elemento predominante, eurocentrista, anglosajón, imperialista, capitalista; hacia un sistema de flujos interconectados junto con la economía, la política, la cultura, aspectos sociales, entre otros. Por tanto, dentro de la academia es necesario replantearse el rol que cumple la arquitectura y el modelo de enseñanza-aprendizaje, y así, construir nuevas metodologías, epistemologías e incluso revisar el lenguaje de las formas; lo que implica reflexionar sobre la influencia racionalista en la concepción del mundo, para construir las nociones de actuación, plantear una nueva posición del arquitecto y la academia.

La complejidad se puede entender como un vacío conceptual en la disciplina de la arquitectura, reflejo de la falta de pensamiento crítico latinoamericano, que, sin importar las diferencias geográficas, ideológicas, culturales, sociales y económicas, se reproducen objetos arquitectónicos tardorracionalistas y especulativos, como si fuesen un apéndice del pensamiento foráneo generalista de las principales metrópolis globales. Sistemas urbanos y arquitectónicos están expuestos a masivas, libres y espontaneas circulaciones de flujos entrópicos que sobrepasan la conciencia humana, formas complejas y seductoras, sin límites, diseñadas para una sociedad del consumo que reconoce lo objetivo y se olvida de lo subjetivo, es decir, del ser humano.

Por tanto, desde la Facultad de Arquitectura y Urbanismo de la Universidad Central del Ecuador (FAU-UCE), el reto empieza por vislumbrar la metamorfosis del proceso de formación en el arquitecto, que incluye las metodologías utilizadas, la demanda de la sociedad y del mercado, la práctica, teoría y crítica arquitectónica, los modos de producción de la ciudad, el cambio tecnológico, la crisis ambiental, la identidad local, que en suma, llegan a cuestionar la pertinencia de la Carrera de Arquitectura y el perfil profesional que demandan respuestas actuales del campo disciplinar. Acoger el paradigma de la complejidad dentro de los procesos de la enseñanza-aprendizaje en la arquitectura y urbanismo, sirve de aporte en la construcción colaborativa y participativa de una nueva Reforma Académica en la FAU dentro de la Institución y la planta docente. 


\section{LA COMPLEJIDAD SISTÉMICA EN LA CIUDAD Y LA ARQUITECTURA}

La arquitectura de la ciudad latinoamericana atraviesa una nueva etapa de la economía-mundial (Ianni, 1996), que implica la nodalización de las cadenas productivas a través de objetos arquitectónicos que enlazan flujos de centralidad con lugares descentralizados en una red urbana global compuesta de centros y periferias, en escalas grandes y pequeñas, comportándose como un sistema complejo, a lo que Dussel (2018) denomina sistema-mundo. Esta noción de sistema conforma las diversas relaciones nacionales, internacionales, transnacionales, mundiales y globales en interacción con actores sociales, institucionales, empresariales o corporativos con capacidad de decisión sincrónica en beneficio de los intereses hegemónicos.

Razón por la cual, el nuevo paradigma de la complejidad involucra el desafío de la interdisciplinariedad, donde la arquitectura debe ser atendida desde muchos aspectos: el ámbito social, ambiental, ecológico y el aspecto económico; esta multiplicidad de enfoques permite una visión más clara de la realidad. A partir de los años 70 se plantea una revisión crítica de la arquitectura a nivel mundial que acepta la complejidad y la heterogeneidad (Maluenda, 2016), con múltiples sistemas dinámicos simultáneos.

Bajo este aspecto, Penrose (2006) hace un recuento de los cambios de pensamiento que han renovado esta visión de la realidad, es así que, durante el siglo XIX el pensamiento sistémico de Kant, Hegel, Marx y otros, abrió la posibilidad de subdividir el todo en partes que se relacionan según sus características, sin embargo, era un proceso lineal en busca de homogeneizar el conjunto de elementos. A mediados del siglo XX, este paradigma cambió hacia un proceso de interrelaciones al demostrar que, dentro de los conjuntos, existen otras relaciones dependientes o independientes como un todo organizado. De esta visión sistémica surgen teorías como: "la teoría de la relatividad de Einstein, la teoría del campo de Kurt Lewin, la teoría de la Gestalt de Wertheimer, Kohler y Koffka, la teoría de la comunicación de Shannon y Weaver, la teoría de las redes de Rapoport, la teoría de la cibernética de Norbert Wiener y la teoría general de los sistemas de Bertalanffy, entre otras.” (Bermeo, 2019, pág. 182).

Como resultado, en el sistema, los conjuntos no pueden ser isomorfos a la realidad, sino solo entre sí (Von Bertalanffy, 1993), entonces, en el campo de la arquitectura, los objetos arquitectónicos son diferentes entre sí, pero siempre deben ser fieles a la realidad existente y estar supeditados a interactuar con sujetos, quienes los habitan para trabajar en sinergia como un conjunto. La variable sujetos, dio paso a la teoría de los sistemas sociales donde Niklas Luhmann (1998) considera a los sujetos como sistemas psíquicos y sus cuerpos como sistemas orgánicos. Así en el siglo XXI, ya no es suficiente la razón y su predominio, aparecen interrogantes debido a las dicotomías en el conocimiento entre los elementos objetivos y subjetivos (Barrios, 1997). Autores como Habermas (1989) plantean alternativas que proponen unir el aspecto afectivo y el racional en el conocimiento, entonces, dentro del análisis se suma lo emocional, lo subjetivo y lo espiritual como parte fundamental de la disciplina de la arquitectura.

Es decir, se debe superar al racionalismo, que está apoyado en el pensamiento totalitario de Aristóteles y la realidad fragmentada de Descartes, que hacen referencia al paradigma de la máquina. Es muy evidente cómo la idealidad platónica está presente en 
las ideas lecorbusieranas y genera confusiones, por ejemplo, la idea de naturaleza concebida como algo racionalizado, humanizado o conquistado; lo que en la actualidad cambia, y se concibe al hombre como parte de la naturaleza, no como dueño de ella, donde los sujetos son seres autopoiéticos (Maturana \& Varela, 2006), porque se autoorganizan en fronteras y su interacción en el medio ambiente los hace ser sociales, cumpliendo con la propiedad sistémica de acoplamiento estructural.

En la complejidad existen múltiples interconexiones, con la propuesta sistémica según Bertalanffy (1993) se articulan tres escalas: sistemas, subsistemas y suprasistemas, del mismo modo, la teoría sistémica Luhmanniana (1998) se escala dependiendo su interacción, organización y lo social. Por un lado, está la realidad de la naturaleza, y por otro lado el conocimiento real, como algo que existe entre la naturaleza con sus construcciones culturales y emocionales; así, lo que se consideraba como un universo totalitario, ordenado y constante, ahora es fragmentado, indefinido y caótico. En este panorama, la arquitectura del siglo XXI debe establecer su propio sistema arquitectónico autopoiético capaz de resistir y sobrevivir a los ataques de la globalización, la máquina y sus efectos en lo urbano y lo social.

\section{SUBJETIVIDAD, OBJETIVIDAD Y ESPACIALIDAD DENTRO DE LA ARQUITECTURA}

El poder sistémico de la ciudad hegemónica ejerce sus interrelaciones sobre las ciudades subordinadas a través de la búsqueda de patrones de comportamientos sociales como creadores de vida. Dentro del campo de la arquitectura, ya Alexander (2004) proponía la posibilidad de edificar en un medio ambiente ordenado y coherente mediante piezas entrelazadas perfectamente, así, como lenguaje crea elementos con cualidades llamados patrones, que comprenden los modos intemporales de construir, vivir y reflejar una certeza intuitiva de cómo funciona la realidad en un todo ordenado.

Sin embargo, la complejidad evidencia que un sistema es autopoiético, necesita de organismos vivos, de sus interrelaciones de poder y de sus series reproductivas, donde cada uno se distinga por su singularidad o pattern, espontaneidad, diversidad y variabilidad, conformando un sistema social de autoproducción de lo emergente en medio de un caos (Maturana \& Varela, 2006). Por consiguiente, los principios estereotipados de la arquitectura moderna construyeron ciudades con edificaciones miméticas a los centros de poder, a las que critica Jacobs (2016), porque cayeron en la monotonía y la degradación de los espacios públicos. Añade Alexander (2016), que son ciudades superpuestas a las estructuras del pasado, sin principios ordenadores por haberse limitado solo a reproducirlas. Esto resalta la importancia de la interrelación que deben tener las partes en un sistema, pues en la complejidad, la estética que adopta un sistema depende de su filogenia y ontogenia.

En los años 50 ya son muy evidentes las reacciones en contra de los ideales de la modernidad, y la crítica a la Carta de Atenas promueve una reflexión hacia las ciudades tradicionales. Las ciencias sociales se preocupan de las relaciones entre centro y periferia, analizan temas en cuanto a lo tradicional y moderno, en medio de un contexto de deslocalización industrial, urbanizaciones difusas que proliferan en las periferias, vacíos 
urbanos en zonas compactas, lugares abandonados, exclusión y desigualdad social; prácticamente se marca el fracaso de la modernidad en la ciudad (Gorelik, 1999).

La década de los 60 en Latinoamérica, es un periodo de cambio que permite entender la complejidad y diversidad de los años precedentes, para Marina Waismann (1989) se debe replantear el problema de ideología y analizar el contexto existente; a partir de este momento ya no se debe leer de forma lineal los procesos históricos, más bien, se debe conformar un sistema complejo donde intervengan otras disciplinas como es la antropología, la sociología, la semiología, la política, etc. Por lo tanto, la arquitectura debe apoyarse en el análisis del objeto y su entorno, que permitan entender el por qué se da la composición arquitectónica según su forma, estructura o la red de relaciones entre ellos con el contexto geocultural para así marcar lineamientos hacia el futuro.

A partir de los años 80 la arquitectura busca mantener un lenguaje universal pero haciendo referencia a elementos simbólicos iconográficos, donde se retoma el discurso de abstracción y permite una traducción formal de elementos compositivos que pueden derivarse de la arquitectura tradicional, a esta etapa se la reconoce como posmoderna, que contiene un discurso compositivo y de significación, esto quiere decir que la hibridación presenta un riguroso manejo de la forma que aborda la complejidad como una necesidad dentro de la creación arquitectónica, entonces cada lugar tiene una forma particular de expresarse.

En la actualidad la crítica arquitectónica no es la que propone regresar a las formas del pasado preindustrial, más bien lo que se intenta es acoger la tecnología de avanzada, pero sin dejar de lado elementos históricos; el resultado sería una arquitectura local con toques de técnica universal. En Latinoamérica se han dado varias interrupciones en los procesos históricos, que se identifican con hechos puntuales, uno de ellos es la influencia de la cultura occidental y la apertura a la globalización; entonces se puede asumir que aparecen momentos de transición, que Roberto Fernández (1998) los denomina fases de hibridación, las mismas que influyen en la producción material arquitectónica.

García Canclini (1975) se refiere a estos fragmentos como el resultado de distintas etapas del desarrollo de la ciudad, donde pueden ocurrir conflictos por la contigüidad de las construcciones, por la diferencia de organización de los espacios, o por los comportamientos espaciales. Todos estos factores viven en coexistencia de la diversidad en una misma ciudad y son una respuesta más a las manifestaciones de la época. Estas interrelaciones internas o externas forman sistemas de objetos (Baudrillard, 2016) que nacen de la necesidad del sujeto para mejorar su calidad de vida, esto permite la construcción de ciudades más flexibles, incluyentes, tolerantes, equilibradas, dialógicas y democráticas, a manera de ciudad collage (Rowe, 2013). Además, amplían las formas de comunicación al identificar patrones identitarios como "lo trivial y la belleza de lo ordinario" (Venturi, 2016, pág. 132) que permiten vincular el sistema social al sistema arquitectónico.

Aquí entra en reflexión las diferencias entre hábitat y habitar; dentro del habitar interviene la cultura, que comprende cómo el sujeto se vincula con la realidad, reconociendo objetos que se encuentran en el entorno para "ubicarse", donde el simple hecho de reconocer un espacio, es apropiarse de él, y es lo que da sentido a la ubicación 
del sujeto, y del sujeto frente a los demás; lo que Ángela Giglia llama “establecer presencia con respecto a un entorno espacial" (2007, pág. 5).

El hecho de habitar es relacionarse con el mundo, después de transformar un espacio con una intervención humana, es un proceso de interpretación continuo, de modificación, de simbolización, por lo tanto, de intervención cultural. La arquitectura al ser una disciplina necesita apoyarse de otras ciencias y disciplinas para aportar en el conocimiento, por ejemplo, en las ciencias sociales, mediante la teoría del babitus donde Pierre Bourdieu (1980) explica cómo el sujeto se moldea según las condiciones materiales y culturales del mundo, el hecho de hablar, de caminar, de sentir, de pensar, de ser; se contrasta con aspectos subjetivos que se reflejan en vínculos de las estructuras sociales, y se lo debe entender desde las prácticas individuales y colectivas como patrones reiterativos en los comportamientos del sujeto a lo largo de la historia.

Incluir la subjetividad como la relación sujeto-objeto, es algo que el racionalismo no reconoce; el mirar con otros enfoques a la arquitectura permite la comprensión de cada espacio, teniendo en cuenta que el sujeto necesita construir su propio hábitat (Rapoport, 1972). Dentro de la Arquitectura se debe incluir el análisis de un sistema social, una familia o vecindario, mediante la conceptualización del espacio vivido y construido que refleje una realidad. Se necesita también de la conceptualización del lugar, por lo tanto, se establece un marco teórico referencial que se aplica a la forma del habitar social (Sepúlveda, 1991), así, los sujetos-objetos se construyen, modifican, adaptan constantemente en una ciudad abierta (Sennet, 2015) siempre incompleta e inesperada.

\section{LA ENSEÑANZA INTERDISCIPLINAR EN LA FAU: UNA COMPLEJIDAD DESDE EL SUR}

La FAU-UCE a lo largo de sus 62 años (1959-2021) de vida institucional académica, ha sido partícipe de la producción urbana y arquitectónica principalmente de las ciudades ecuatorianas, atravesando 9 reformas al Plan Académico y Mallas Curriculares (19462017). En la última reforma a la malla se plantea el trabajo interdisciplinario con criterios y organizaciones curriculares de forma horizontal y vertical, como una matriz de doble entrada que resalta en cada nivel o semestre un resultado de aprendizaje, donde pueden colaborar varios campos del saber que se complementan entre sí. Dicha reestructuración es la respuesta a los cambios evidentes que afrontan las dinámicas urbanas en el contexto de la complejidad y los sistemas, que han provocado como menciona Piñón (2005) una pérdida de teoría de la arquitectura, produciendo en los objetos arquitectónicos una vulgaridad figurativa y en la sociedad una cultura del espectáculo, despilfarro y consumo, incentivado o ejecutado por el poder económico especulativo de las inmobiliarias y arquitecturas comerciales.

Esta problemática obliga ampliar la visión de la FAU-UCE hacia un pensamiento complejo y sistémico sobre la enseñanza de la arquitectura y sobre la arquitectura en sí, con un enfoque latinoamericanista para recuperar valores y necesidades de la gente. La Facultad ha heredado la arquitectura racionalista, manteniendo un proceso de enseñanza basado en el pensamiento lógico, es decir, procesos ordenados por etapas como: análisis, síntesis y diseño, que al término de cada una producen un corte al conocimiento, siendo el estudiante incapaz de correlacionar la parte reflexiva de la proyectual. "Bajo esta 
premisa, se ve la necesidad de buscar la integración de los conocimientos de forma simultánea, dejando el proceso lineal-tradicional para adoptar un proceso complejo que recoja todas las variables de creación, reflexión e intuición” (Bermeo, 2020, pág. 1).

En otras palabras, la FAU-UCE debe partir de la necesidad de crear una cultura arquitectónica local, como elemento de las complejas relaciones de producción urbana, social y económica, y, al ser una Universidad Pública de Latinoamérica, las reflexiones provienen desde las epistemologías del Sur. Si bien existe un papel hegemónico de la episteme occidental que no se puede negar, es necesario reflexionar sobre Latinoamérica que tiene un pensamiento diferente a la modernidad global que se articula con la identidad. Este debate abre posibilidades de estudio a realidades diferentes y poco exploradas que niegan el universalismo, lo que Sousa Santos (2003) llama línea abismal, refiriéndose a la negativa sobre las experiencias y manifestaciones no-occidentales, las cuales siempre son minimizadas o se los considera desechos culturales.

Cabe preguntarse: ¿Los países en vías de desarrollo pueden aplicar las políticas de los países desarrollados? Cuando se sabe que Latinoamérica se caracteriza por lo popular e informal (Canolly, 2013). La enseñanza-aprendizaje en la FAU-UCE debe alcanzar un mestizaje académico como una forma de resistencia a la ciudad burguesa, para que el hábitat y el habitar perduren con rasgos locales, a pesar de la imposición de modelos que provienen de Occidente (Fernandez, 1998). El sur, tiene culturas subordinadas, imaginarios dominantes, con la tendencia de homogenizar a través de la moda, el sur imita las modas por la globalización de las culturas (Quijano, 2000). La FAU-UCE debe aprender a existir en un sur donde la modernidad llegó y sufrió un proceso de hibridación, y aceptar un mundo plural, complejo, diverso, sin jerarquías interculturales (García, 2001).

La FAU-UCE al acoger los códigos arquitectónicos de la modernidad, actúa de forma contradictoria con la dinámica de la vida, ya que muchos estudiantes provienen de territorios rurales y formas de vida propias; además, las diferencias tecnológicas en una zona poco desarrollada es la mayor dificultad para que se cumplan los ideales de la modernidad. En América Latina la arquitectura refleja una modernización caracterizada por la interrelación de modelos importados y factores locales que le otorgan rasgos particulares, nuevos valores, nuevas culturas (Cárdenas, 1996). La producción arquitectónica desde la academia debe dar importancia al lugar, ese es el valor que tiene la arquitectura, los límites dependen de la gente y es para la gente que se hace arquitectura (Frampton, 1981).

Además, la crítica a la arquitectura moderna debe surgir para promover nuevas epistemologías que aparecen con la complejidad, donde se considere a los otros y al pensamiento del otro (Mignolo, 2000). Esa revalorización no solo es desde las escuelas de arquitectura, sino también, desde lo político, económico, social, que conllevó a muchos países a crear nuevas Constituciones como: Bolivia en 2007 y Ecuador en 2008, que promovieron la revalorización de territorios originarios y la noción del Ayllu o comunidad, así como, el Suma Kawsay o buen vivir respectivamente. Estos ejemplos demuestran la recuperación de identidad que aporta una alternativa frente a las contradicciones de la racionalidad occidental. Plantear una descolonización mental tanto a docentes, estudiantes y plantearlo dentro de la malla curricular, es vivir un bagaje cultural 
que Europa no lo tiene, dejar de imitar a los estereotipos occidentales incluso la estetización de la pobreza que se ha convertido en moda.

Era inevitable, que la FAU-UCE al igual que pasó en todas las facultades de Latinoamérica, se acojan el discurso de la modernidad y se exprese a través de la ciudad, porque para varios arquitectos ese modelo moderno da un nuevo sentido a la ciudad (Arango, 2014); pero, los procesos de modernización en Latinoamérica también son actos colonizadores que tienen consecuencias negativas a nivel social, además que, después de años de crítica se puede evidenciar sus limitaciones.

Dentro de la malla curricular de la FAU-UCE, se declara como campo epistemológico a la complejidad, y además, la creación de la cátedra integradora permite la conceptualización de la teoría de sistemas arquitectónicos, donde se puede responder a un proyecto compartido por sujetos en una cultura, y se visibiliza como objeto a los sistemas de objetos interconectados con el ser humano, en forma sinestésica e intramodal, encargados de proyectar la dimensión del espacio vivido y de la esencia del espacio construido (González, 2014). Además, la catedra integradora permite un trabajo complementario con las demás asignaturas con lo que se conforma el proyecto integrador; la arquitectura debe estudiarse desde varios ejes, pero estos ejes no pueden tratarse por separado, esta interdisciplinariedad se la declara desde los primeros niveles y con más énfasis en los últimos. Así como, se incluye el trabajo de titulación, donde se ha obtenido buenos resultados por realizar estudios interdisciplinarios al momento de abordar el proyecto arquitectónico o urbano.

La FAU-UCE debe dejar la arquitectura de reproducción o estandarización, que rechaza la investigación científica; de no ser así, el proyecto sigue siendo un tipo de arquitectura, una estructura genética, visto como repetición y establecido como un modelo de construcción intelectual en sí misma (Martín, 1984). Más bien se propone una dialéctica con la historia, teoría de la arquitectura, ciencias sociales y con otras disciplinas, interdisciplinas y transdisciplinas. La integración de otras Facultades podría fortalecer la carrera de arquitectura, mientras no se pierda el fin, la arquitectura, de hecho, la transdisciplinariedad marca inevitablemente la crisis del hedonismo académico o de la posición dominante del docente, tal vez de esta manera, se pueda recuperar el oficio de la arquitectura, así como también, dar paso a la conformación de nuevas carreras dentro de la FAU-UCE.

Esta visión sistémica de la enseñanza-aprendizaje de la arquitectura debe incluir también, la práctica sobre el territorio a través de programas de vinculación con la sociedad que concienticen al estudiante sobre la realidad social, económica y cultural, integradas a proyectos de investigación a través de laboratorios, observatorios y grupos de investigación. Pero también, el anclaje entre pregrado y posgrado es importante para la formación académica estudiantil y docente, que permita, además, ser la vOz académica en las decisiones de las políticas públicas de las ciudades y de los gobernantes, solo así la academia tal como la arquitectura y las ciudades conformarán una estructura de semientramado que interconecte todos los sistemas complejos del siglo XXI. 


\section{CONCLUSIONES EL PENSAMIENTO COMPLEJO EN LA ENSEÑANZA DE LA ARQUITECTURA}

Tradicionalmente los arquitectos han percibido los problemas arquitectónicos fuera de las realidades complejas, hoy se entiende que todo lo que conforma la arquitectura debe interactuar entre sí, de tal forma, que la tecnología constructiva, la teoría, la crítica, y la expresión gráfica, se relaciona juntamente con el proyecto arquitectónico, y a su vez con el barrio y ambos con la ciudad. Hoy no se puede entender la arquitectura sin la ciudad y la ciudad sin la arquitectura, más aún, se entiende que estos deben relacionarse con otros campos como es: el productivo, económico, social, ambiental, cultural, etc., pues se debe interactuar con otros actores y profesiones.

La arquitectura debe recuperar el arte y la técnica del oficio a través de la teoría y la praxis que re-construyan el concepto del proyecto, un proyecto que contenga el espíritu del lugar, que se relacione con el contexto glocal, que sea incluyente con el otro y abierto a la alteridad, un proyecto que sea mucho más que un estilo, forma o función, un espacio vivido decretado por lo social, lo común y lo público, lejos de cualquier espacio mercantilizado y banal.

La arquitectura tiene un usuario con características particulares reales, que no son las mismas del imaginario del arquitecto, por lo tanto, comenzar a fortalecer la relación y participación ciudadana como hecho primordial dentro de la práctica disciplinar. Una posibilidad es regresar los ojos hacia la realidad social, compuesta por múltiples componentes a diferentes escalas. En este espacio se puede tomar conciencia de la crisis en la que vivimos, como es la limitación de recursos naturales, la necesidad de fortalecer el diálogo de saberes como nuevas formas de conocimiento y palpar la realidad desde un contexto latinoamericano que permita entender las respuestas en el campo de la arquitectura sin necesidad de imitar modelos euro centristas.

Abordar la complejidad de la arquitectura es: por un lado, responder a la realidad de la naturaleza donde existen múltiples interconexiones; y por otro lado al conocimiento real, como algo que existe entre la naturaleza con sus construcciones culturales y emocionales.

\section{REFERENCIAS BIBLIOGRÁFICAS}

Alexander, C. (2004). A Vision og a Living World: Nature of Order. Berkeley: Center for Environmental Structure.

Alexander, C. (2016). La ciudad no es un árbol. Barcelona: Escuela Técnica Superior de Arquitectura de Barcelona. Sección Publicaciones.

Arango, S. (2014). Ciudad y Arquitectura. Seis generaciones que construyeron la America Latina Moderna. México: Fondo de Cultura Económica.

Barrios, D. M. (1997). La crisis del pensamiento moderno. Memorias del seminario de perfiles profesionales. Monterrey: UANL.

Baudrillard, J. (2016). El sistema de los objetos. Buenos Aires: Siglo XXI.

Bermeo, S. (2019). Arquitectura de Flujos: El metasistema arquitectónico tardorracionalista en la hipermodernidad de las ciudades globales latinoamericanas. 
Tesis Doctoral. Guadalajara: Universidad de Guadalajara - Centro Universitario de Arte, Arquitectura y Diseño.

Bermeo, S. (2020). El Modulor: Positivo para COVID-19. FAU Editorial, 1-11.

Borja, J. D. (2003). La ciudad conquistada. Madrid: Alianza editorial.

Bourdieu, P. (1980). El sentido práctico. Madrid: Taurus.

Canolly, P. (2013). La ciudad y el hábitat popular: paradigma latinoamericano. Teorías sobre la ciudad en América Latina. Ciudad de México: Universidad Autónoma Metropolitana.

Cárdenas, E. (1996). Valorar la arquitectura desde la óptica de la identidad cultura. La Habana: Instituto Superior Politécnico José Antonio Echeverría.

de Mattos, C. A. (1998). Reestructuración, crecimiento y expansión metropolitana en las economías emergentes latinoamericanas. Economía, Sociedad y Territorio 1(4), 723-753.

Dussel, E. (2018). Filosofía de la liberación. México: Fondo de Cultura Económica.

Fernandez, R. (1998). El laboratorio americano. Madrid: Biblioteca Nueva.

Frampton, K. (1981). Historia crítica de la arquitectura moderna. Barcelona: Gustavo Gili.

García, N. (2001). Culturas híbridas. Estrategias para entrar y salir en la modernidad. Buenos Aires: Paidos Ibérica.

Giglia, Á. (2007). El habitar y la cultura. México: Universidad Nacional Autónoma de México.

González, A. A. (2014). De la educación en arquitectura. México D.F.: Universidad Iberoamericana.

Gorelik, A. (1999). Ciudad, modernidad, modernización. Imagen de la modernidad. Bello Horizonte: Auténtica.

Habermas, J. (1989). El discurso filosófico de la modernidad. Buenos Aires: Taurus.

Ianni, O. (1996). Teorías de la globalización. México: Siglo XXI.

Jacobs, J. (2016). The death and life of great American cities. New York: Vintage.

Luhmann, N. (1998). Sistemas sociales: lineamientos para una teoría general. México: Anthropos editorial.

Maluenda, E. (2016). La arquitectura moderna en Latinoamérica. Antología de autores, obras y textos. Barcelona: Reverté.

Martín, M. (1984). La tipología en la Arquitectura (Doctoral dissertation). Las Palmas de Gran Canaria: Universidad de las Palmas de Gran Canaria.

Maturana, H., \& Varela, F. (2006). De máquinas y seres vivos: autopoiesis: la organización de lo vivo. Santiago de Chile: Editorial Universitaria.

Mignolo, W. (2000). Local Histories/Global Designs: Essays on the Coloniality of Power, Subaltern Knowledges and Border Thinking. Princeton: Princeton University Press. 
Morin, E. (2004). La epistemología de la complejidad. Gazeta de Antropología, 1-13.

Norberg-Schultz, C. (1975). Existencia, espacio y arquitectura. Barcelona: G. Gili.

Parnreiter, C. (2005). Tendencias de desarrollo en las metrópolis latinoamericanas en la era de la globalización: los casos de Ciudad de México y Santiago de Chile. EURE (Santiago), 31(92), 05-28.

Penrose, R. (2006). Lo grande, lo pequeño y la mente humana. Madrid: Akal.

Piñón, H. (2005). La forma y la mirada. Cataluña: Nobuko.

Quijano, A. (2000). Quijano, Aníbal 2000 "Colonialidad del poder, eurocentrismo y América Latina" en LandeLa colonialidad del saber: eurocentrismo y ciencias sociales. Buenos Aires: Clacso.

Rapoport, A. (1972). Vivienda y cultura. Barcelona: G. Gigli.

Rowe, C. \&. (2013). Collage City. In The Urban Design Reader. New York: Routledge.

Santos, B. d. (2003). Crítica de la razón indolente. Bilbao: Desclée de Brouwer.

Sassen, S. (1994). Global city. New York, London, Tokyo. New Jersey: Princeton University Press.

Sennet, R. \&. (2015). La ciudad abierta. Bogotá: interacciones urbanas y movilidad futura (págs. 1-16). Bogotá: Pontificia Universidad Javeriana.

Sepúlveda, R. (1991). Progresividad Habitacional. Un estudio Sociofísico del programa de mejoramiento de Barrios. Santiago, Chile: Instituto de Vivienda del a Facultad de Arquitectura y Urbanismo y el departamento de Sociologia de la Facultad de Ciencias Sociales.

Venturi, R. I. (2016). Aprendiendo de las Vegas. Barcelona: Gustavo Gil.

Von Bertalanffy, L. (1993). Teoría general de los sistemas. México: Fondo de cultura económica.

Waismann, M. (1989). Las corrientes posmodernas vistas desde América Latina. Summa $\left(\mathrm{N}^{\circ} 260\right) \cdot \mathrm{pp} .44-47$. 